\title{
Detection of a companion to the pulsating sdB Feige $48^{\star}, \star \star$
}

\author{
S. J. O'Toole ${ }^{1}$, U. Heber ${ }^{1}$, and R. A. Benjamin ${ }^{2}$ \\ ${ }^{1}$ Dr Remeis-Sternwarte, Astronomisches Institut der Universität Erlangen-Nürnberg, Sternwartstr. 7, \\ Bamberg 96049, Germany \\ e-mail: otoole@sternwarte.uni-erlangen.de \\ 2 Department of Physics, University of Wisconsin-Whitewater, Whitewater, WI 53190, USA
}

Received 13 February 2004 / Accepted 30 April 2004

\begin{abstract}
We present the discovery of a binary companion to the pulsating sdB Feige 48. Using HST/STIS and archival FUSE spectra, we measure a period of $0.376 \pm 0.003 \mathrm{~d}$ and a velocity semi-amplitude of $28.0 \pm 0.2 \mathrm{~km} \mathrm{~s}^{-1}$. This implies that the companion star must either be of very low mass, or the orbit is at low inclination. Combining $2 M A S S$ fluxes, the lack of a reflection effect, results from asteroseismology and a measurement of the rotation velocity of Feige 48, we show that the orbital inclination must be $\leq 11.4^{\circ}$ and that the unseen companion is a white dwarf with mass $\geq 0.46 M_{\odot}$. The implications of this discovery, and of binarity amongst sdB pulsators, is then discussed in the context of recent theoretical work on sdB formation. In particular we suggest that radial velocity studies focus on sdB pulsators with no known companion, and that asteroseismological studies of sdBs investigate a larger mass range than previously considered in order to test formation models.
\end{abstract}

Key words. stars: individual: Feige 48 - stars: binaries: close - stars: oscillations

\section{Introduction}

In recent years, interest in hot subdwarf B (sdB) stars has increased dramatically. This is in large part due to the discovery of rapid oscillations in some sdBs, but also because improving instrumentation has allowed more detailed studies of their spectral properties. It is now generally accepted that sdBs are associated with stars on the extreme Horizontal Branch (EHB) (e.g., Heber 1986; Saffer et al. 1994). This means they are low-mass stars $\left(\sim 0.5 M_{\odot}\right)$ that burn helium in their cores and have hydrogen envelopes too thin $\left(<0.02 M_{\odot}\right)$ to sustain nuclear burning. Stellar evolution calculations by Dorman et al. (1993) predict that because of their thin envelopes, sdBs will evolve directly to the white dwarf cooling track, bypassing the Asymptotic Giant Branch.

While the next stages of sdB evolution appear to be known, the question of sdB formation remains unanswered. There have been three basic scenarios proposed: binary interaction involving Roche lobe overflow (Mengel et al. 1976), single star evolution with strong mass loss near the tip of the Red Giant Branch (D'Cruz et al. 1996), and the merger of two helium white dwarfs (Iben \& Tutukov 1986). Significant progess has

* Based on observations made with the NASA/ESA Hubble Space Telescope, which is operated by the Association of Universities for Research in Astronomy, Inc., under NASA contract NAS 5-26555. These observations are associated with program \#8635.

$\star \star$ Based on observations made with the NASA-CNES-CSA Far Ultraviolet Spectroscopic Explorer. FUSE is operated for NASA by the Johns Hopkins University under NASA contract NAS 5-32985. been made with the recent work of Han et al. (2002, 2003), who studied three possible binary formation channels: common envelope ejection, stable Roche lobe overflow and the merger of two helium white dwarfs. Using binary population synthesis, they created 12 simulations and found one of them could satifactorily reproduce the observed characteristics of $\mathrm{sdBs}\left(T_{\mathrm{eff}}\right.$, $\log g$, binary fraction, orbital period distribution, etc) when compared to radial velocity studies by Maxted et al. (2001) and Morales-Rueda et al. (2003). Napiwotzki et al. (2004) found a binary fraction of 39\%, lower than predicted, while Lisker et al. (2004), in the largest homogenous study so far, compared their data to the simulations of Han et al. (2003) and found a different best-fit model.

The oscillations detected in some sdBs offer a way to test these formation models. Importantly, pulsations are seen in sdBs with short- and long-period binary companions, as well as in apparently single sdBs. The properties of the V361 Hya stars (the short-period, $p$-mode sdB pulsators, originally called EC 14026 stars) are reviewed by Kilkenny (2002), while Green et al. (2003) presents the detection of long-period, likely $g$-mode pulsations in somewhat cooler sdBs (currently known as the PG $1716+426$ or "Betsy" stars).

Pulsations in the V361 Hya star, Feige 48, were first detected by Koen et al. (1998). It is one of the coolest sdB pulsators in its class, with $T_{\text {eff }}=29500 \mathrm{~K}$ and $\log g=5.50$ (Heber et al. 2000), and was discovered to be pulsating with five periods between 344 and $379 \mathrm{~s}$. The oscillation amplitudes show dramatic variation from night to night, which the authors suggested are due to either changes in pulsation power, the 
beating of unresolved modes, or a combination of the two. In a comprehensive follow-up study, Reed et al. (2004) observed Feige 48 over 5 years, and found that the periods measured by Koen et al. (1998) were resolved and that the amplitudes vary by at least $30 \%$. A tentative model match to four out of five detected periods, suggests that Feige 48 rotates with a period around $10 \mathrm{~h}$. The $T_{\text {eff }}$ and $\log g$ of this model were chosen to be consistent with those derived from spectroscopy. Longterm monitoring by Reed et al. also provided an opportunity to measure the phase stability of the pulsations. In particular, they were able to place constraints on the period and mass of a companion to quite high precision, limiting any stellar-mass companion to have a period shorter than 3 days. In another recent study, Charpinet et al. (2003) found a very good model match to all nine periods detected by high $\mathrm{S} / \mathrm{N}$ photometry using the CFHT. They derived a rotational period of $9.58 \mathrm{~h}$, in good agreement with Reed et al.

In this paper we present the detection of binary motion in Feige 48 , an sdB that was generally assumed to be single. We discuss the nature of the companion, and implications of this detection for current theories of $\mathrm{sdB}$ formation and pulsation.

\section{Observations}

Observations with $H S T / S T I S$ were made as part of an ongoing project to measure metal abundances in pulsators and nonpulsators (proposal \#8635, PI: U. Heber). Analysis of these data will be presented in a future paper (see O'Toole et al. 2003, for preliminary results). Once velocity variations were detected in these spectra, we searched both the HST and FUSE archives looking for any other observations. We found two sets of FUSE observations of Feige 48 in the archive, the first (proposal No. B033, PI: G. Fontaine) was a companion project to our HST/STIS observations, and their goal was also to measure metal abundances. The first results from this analysis is presented in Chayer et al. (2003). The second, larger set of data was part of a project to study molecular gas in the interface between the galactic disk and halo (proposal no. C036, PI: R. Benjamin). Results from this investigation will be presented in a forthcoming paper (Benjamin et al. 2004). Fortunately for us, the star used to illuminate the gas cloud under investigation was Feige 48. The list of observations are shown in Table 1, and their description follows below.

\subsection{HST/STIS UV echelle spectra}

We observed 5 sdBs with HST/STIS: three pulsators (Feige 48, PG 1219+534 and PG 1605+072) and two non pulsators (Feige 66 and Ton S-227). The pulsators were all observed in time-tag mode, made possible by the use of the MAMA detectors. This was not originally intended, but was suggested (fortunately) by the time allocation committee. Each star was observed using two medium resolution echelle gratings, E140M and E230M, giving useful wavelength ranges of 1150-1720 and 1630-2370 $\AA$ respectively. The slit length and width used for all exposures was $0.2^{\prime \prime} \times 0.06^{\prime \prime}$, leading to spectral resolutions of $0.017 \AA$ at $1200 \AA$ and $0.020 \AA$ at $1500 \AA$ with the E140M grating and $0.051 \AA$ at $1700 \AA$ and $0.068 \AA$ at $2400 \AA$
Table 1. HST and FUSE observation log for Feige 48.

\begin{tabular}{|c|c|c|c|}
\hline Date & UT & Exp. time (s) & Instrument \\
\hline 2001-03-26 & $18: 57: 38$ & 2662.828 & FUSE \\
\hline 2001-03-26 & $20: 37: 27$ & 2567.906 & FUSE \\
\hline 2001-05-13 & $06: 53: 42$ & 3600.200 & HST/STIS \\
\hline 2001-05-13 & 07:59:32 & 3600.200 & $H S T / S T I S$ \\
\hline 2001-05-13 & 09:09:23 & 3500.199 & $H S T / S T I S$ \\
\hline 2001-05-13 & $10: 13: 33$ & 3511.199 & HST/STIS \\
\hline 2002-04-03 & $19: 52: 36$ & 3856.992 & FUSE \\
\hline 2002-04-03 & $21: 29: 57$ & 4226.070 & FUSE \\
\hline 2002-04-03 & $23: 14: 48$ & 4181.148 & FUSE \\
\hline 2002-04-04 & 00:59:19 & 4264.227 & FUSE \\
\hline 2002-04-04 & $02: 43: 37$ & 4379.312 & FUSE \\
\hline Total: & & 41175.457 & \\
\hline
\end{tabular}

with the E230M grating. All spectra were processed using the time-tag and standard reduction routines in the STSDAs package in IRAF. The orders containing Ly $\alpha$ were excluded, since they are not useful for a radial velocity analysis.

\subsection{FUSE spectra}

In both sets of observations, Feige 48 was observed using the high-throughput $30^{\prime \prime} \times 30^{\prime \prime}$ LWRS aperture on all four spectrograph channels and recorded in time-tag mode. All data were reprocessed by the CalFUSE pipeline software (version 2.4). Of the four channels available, only the LiF2 channel was used, since it contains no Lyman lines which are affected by interstellar absorption and airglow lines from the Earth. The detector also has the highest sensitivity of all onboard FUSE. The dispersion of this channel is $6.7 \mathrm{~m}^{\circ}$ pixel $^{-1}$ and the wavelength range covered is $1086-1182 \AA$. For a description of the detectors and more information on the FUSE mission, see Moos et al. (2000).

\section{Synthetic spectra}

The UV spectra of Feige 48 (and sdBs in general) contain a large number of spectral lines due to iron-group elements $(\mathrm{V}$, $\mathrm{Cr}, \mathrm{Mn}, \mathrm{Fe}, \mathrm{Co}, \mathrm{Ni}$ ), making continuum definition often difficult. The number of lines complicates abundance analyses, but is very advantageous for measuring velocities, since they are very sharp (rotation velocities in sdBs are typically $\leq 5 \mathrm{~km} \mathrm{~s}^{-1}$ ).

As input for our spectrum synthesis, we used a metal lineblanketed LTE model atmosphere with solar metallicity and Kurucz' ATLAS6 Opacity Distribution Functions. The temperature, surface gravity, and helium abundance were set at $T_{\text {eff }}=$ $29500 \mathrm{~K}, \log g=5.50$, and $\log (\mathrm{He} / \mathrm{H})=-2.93$, as determined by Heber et al. (2000).

The spectra were synthesised using Michael Lemke's version of the LINFOR program (originally developed by Holweger, Steffen, and Steenbock at Kiel University). Oscillator strengths were taken from the Kurucz line list, as 


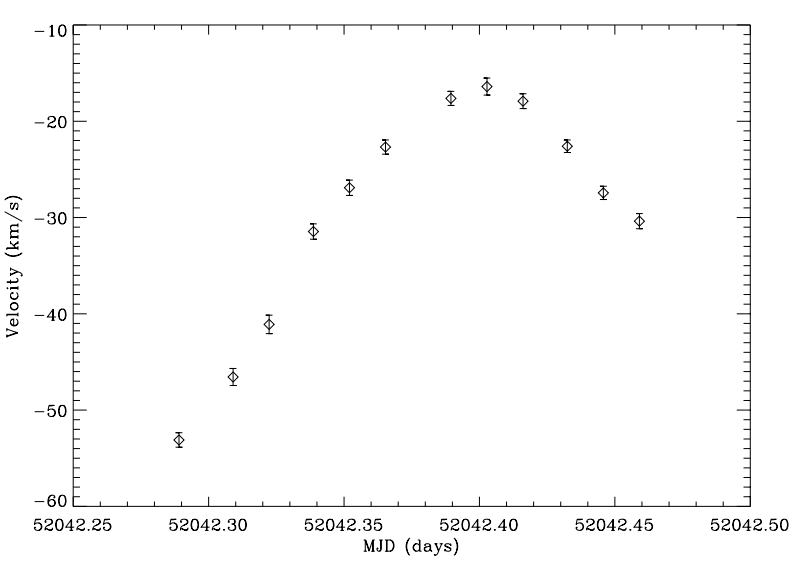

Fig. 1. Velocity variations from far and near UV spectra of Feige 48.

were damping constants for all metal lines. Both the rotation and microturbulent velocities were set to $0 \mathrm{~km} \mathrm{~s}^{-1}$. (The rotation velocity of Feige 48 will be discussed further in Sect. 5).

\section{Binary motion}

During the analysis of abundances in Feige 48, we found that the spectra from the two NUV orbits showed different velocity shifts compared to our synthetic spectra. Because the spectra were taken in time-tag mode, we were able to divide each of the far and near UV exposures into three $1150 \mathrm{~s}$ sub-exposures. These spectra were cross correlated with our synthetic spectra and the results are shown in Fig. 1. The original FUSE observations were also taken in time-tag mode, allowing a similar division, with the exposure time typically being $1050 \mathrm{~s}$.

Using the double-precision cross-correlation routine (rvx) available in $\operatorname{IRAF}^{1}$, we measured the Doppler shift of each newly-created spectrum relative to our synthetic spectra. Since the HST/STIS spectra are in echelle format, each observation yielded 40 velocity measurements (one per order). We calculated the weighted average of these velocities using the inverse square of the velocity errors as weights. The end result of this was a list of velocities with two large gaps in time. These velocities are typically accurate to $\sim 1 \mathrm{~km} \mathrm{~s}^{-1}$.

The analysis of the radial velocity curve is made simpler because the system is single-lined. By fitting the curve with a range of periods we produced a "power spectrum", shown in Fig. 2, which shows the goodness of fit. The actual period described below was found by selecting the highest peak in this spectrum. There is strong aliasing, leading to a relatively large period error (derived by measuring the difference between the nearest alias peaks).

The ephemeris of the system at time $T_{0}$, defined as the conjunction time that the $\mathrm{sdB}$ moves from the blue to red side of the velocity curve, is

$$
\begin{aligned}
\operatorname{HJD}\left(T_{0}\right)= & (2452367.05 \pm 0.03) \\
& +(0.376 \pm 0.0003 \pm 0.003) \times E
\end{aligned}
$$

where the first period error quoted is derived from the uncertainty due to the timespan of the observations, while

\footnotetext{
1 available from http://iraf.noao.edu/extern/
}

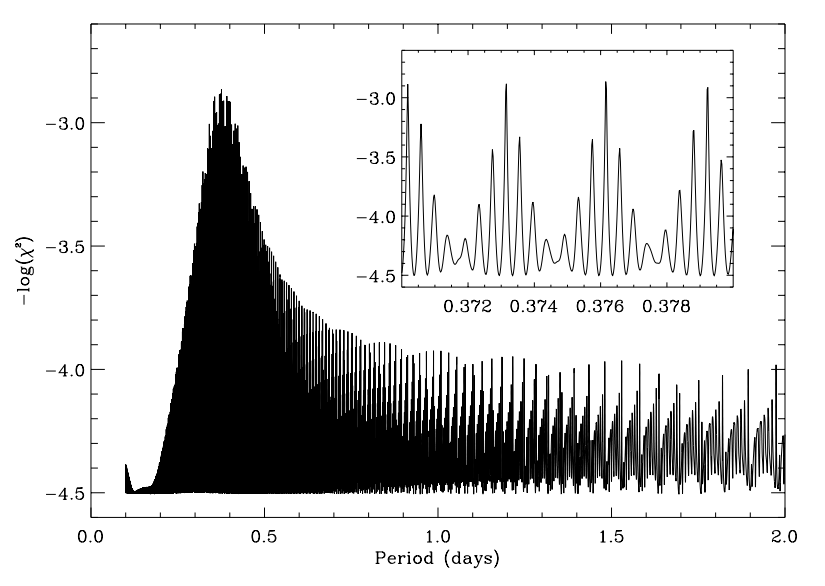

Fig. 2. Amplitude spectrum of the radial velocities of Feige 48. The inset shows the aliasing around the adopted period.

the second error is derived by measuring the difference between the nearest alias peaks, as discussed above. We stress here that these values should not be taken as $1 \sigma$ uncertainties. We measure the velocity semi-amplitude of Feige 48 to be $K_{\mathrm{sdB}}=28.0 \pm 0.2 \mathrm{~km} \mathrm{~s}^{-1}$, while the system velocity is $\gamma=-47.9 \pm 0.1 \mathrm{~km} \mathrm{~s}^{-1}$. The velocity curve phased with this ephemeris is shown in Fig. 3. Despite the separation in time of the observations, the phase coverage is quite good. The period and semi-amplitude of the $\mathrm{sdB}$ can be used to derive the mass function, $f(m)=0.000856 \pm 0.000019 M_{\odot}$. If we assume a mass of $\sim 0.47 M_{\odot}$ for the $\mathrm{sdB}$, as estimated from asteroseismology, then the lower limit for the companion mass is $0.062 M_{\odot}$. Note that the period we have derived here is well below the upper limit set using the phase stability of the pulsations by Reed et al. (2004). Their analysis is insensitive to systems with short periods, small inclinations and massive companions, however, since the light-travel time across the system is far too small to cause a measureable effect on the pulsations.

\section{The nature of the companion}

In order to discuss the implications of this discovery for the formation models of Han et al. (2003), we must first constrain the nature of the companion to Feige 48.

The small mass function implies three possible companion types for Feige 48: a late-type main-sequence star, a brown dwarf or a white dwarf. We can place limits on the first two types using multicolour photometry, as was done by Heber et al. (2003) for the sdB binary HD 188112. Previous studies have suggested that any main sequence companion must have spectral type later than mid K (Koen et al. 1998). We can now compare the $2 M A S S J H K$ magnitudes, along with other Johnson measurements available, with the model spectral energy distribution of Feige 48, shown with photometric measurements overplotted in Fig. 4. Apart from $2 M A S S$, the photometry is obtained from a variety of sources $(U-B=-1.03$, $B-V=-0.25, V=13.48-$ Bern \& Wramdemark 1973, $R=13.57$ (averaged), $I=13.72$ - the USNO-B catalogue, Monet et al. 2003). Magnitudes were converted to fluxes using the calibration of Fukugita et al. (1995) for the optical and Cohen et al. (2003) for the infrared. The model was then scaled 


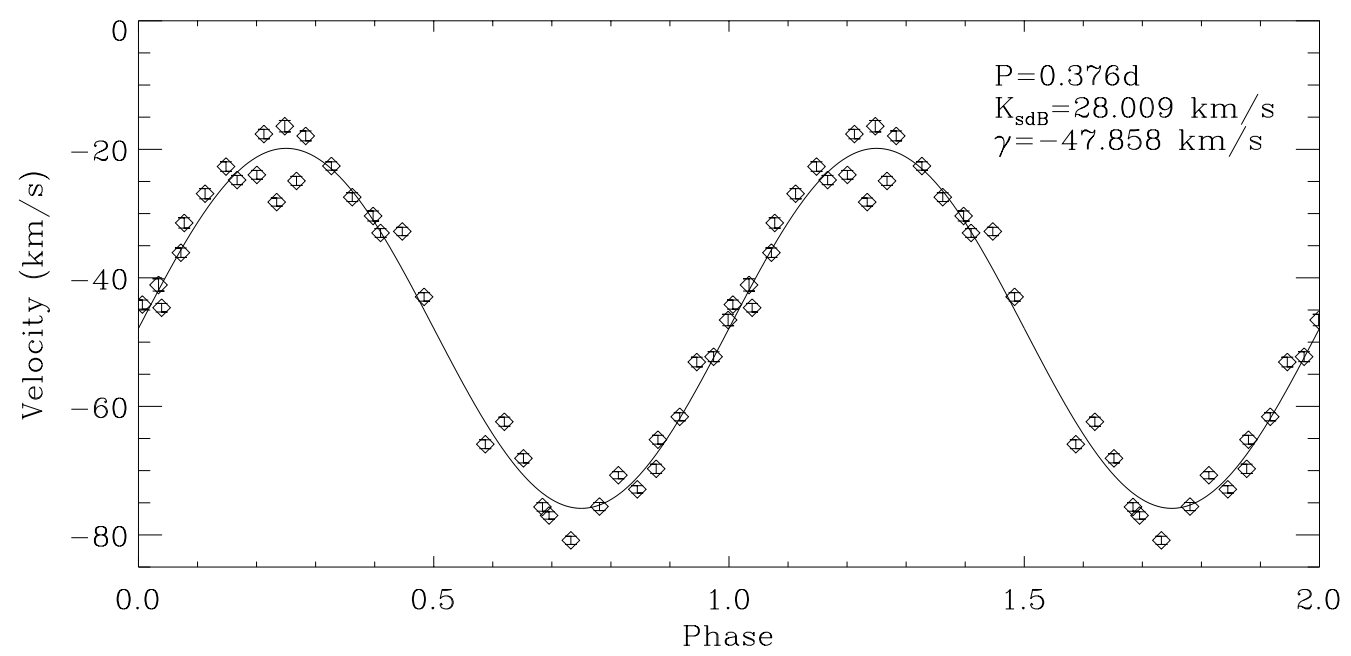

Fig. 3. The phased radial velocity curve of Feige 48. The errors shown are formal errors determined by IRAF.

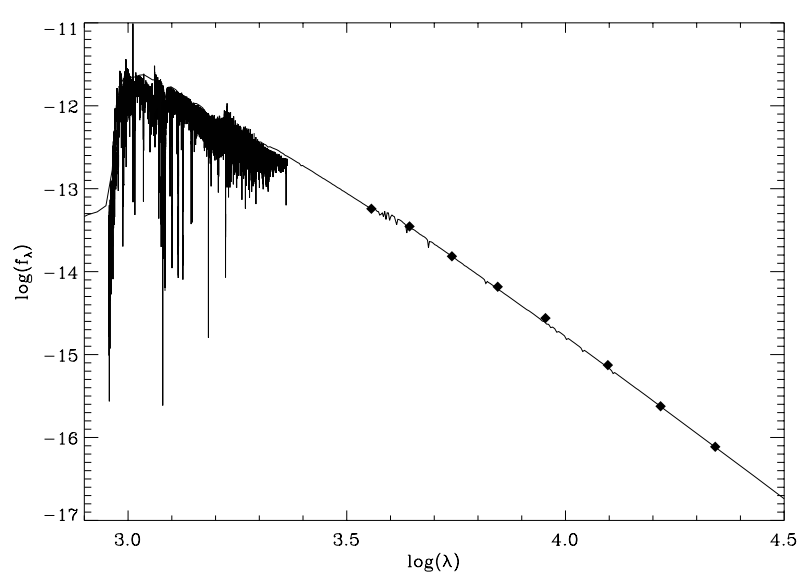

Fig. 4. Spectral energy distribution of Feige 48. The filled diamonds are Johnson magnitudes, including $2 M A S S$ magnitudes. The HST/STIS and FUSE spectra are also shown. There is no evidence for the companion to the sdB.

to the $V$ flux of Feige 48, implying a distance to the system of $755 \pm 90$ pc. As can be seen in Fig. 4, there is no contribution to the flux from the companion in the $K$-band (on this scale, the errors in $J H K$ magnitudes are smaller than the data points). Using the absolute $K$ magnitude of dwarfs of spectral types M 2-M 9 determined by Beuermann et al. (1999), we find that if the companion to Feige 48 is on the main sequence, its spectral type must be M3 or later. This type of star will have a mass of $\sim 0.35 M_{\odot}$, leading to a lower limit for the inclination angle of $\sim 14^{\circ}$.

We can also consider information that has been obtained from the pulsations themselves. Reed (2001), Reed et al. (2004) and Charpinet et al. (2003) have carried out asteroseismological analyses of Feige 48, and suggest a rotation period of $\sim 9.5-10 \mathrm{~h}$. Given the uncertainties involved in the sdB evolution models used and the aliasing seen in Fig. 2, this is in very good agreement with the orbital period of $9.03 \mathrm{~h}$ determined in Sect. 4. In particular, both Reed (2001) and Reed et al. (2004) assumed a rotational splitting coefficient $\left(C_{n l}\right)$ of zero. If $C_{n l}$ is set to 0.1 , which is not unreasonable for a slightly evolved $\mathrm{sdB}$, then the rotation period would be $\sim 9 \mathrm{~h}$.

We now compare the observed upper limit on a reflection effect to what might be expected from an M 3 dwarf with radius $\sim 0.35 R_{\odot}$. A similar system, but with higher inclination and shorter period is the sdB+dM HS 2333+3927 (Heber et al. 2004). In this case a large reflection effect is observed $\left(\Delta V=0.28 \mathrm{mag}\right.$ ) even though the inclination is only $36-39^{\circ}$. This suggest that an effect might be measureable for Feige 48 if present. Calculations by $\mathrm{H}$. Drechsel (private communication) find that an $\mathrm{sdB}+\mathrm{dM}$ system with a period of $9 \mathrm{~h}$ and inclination $15^{\circ}$ would show luminosity variations of $0.025-0.030 \mathrm{mag}$ for maximal albedo of the secondary. The only time-series observations of Feige 48 made were optimised for oscillation studies, not searching for a reflection effect. With an orbital period of $9 \mathrm{~h}$, such an effect is difficult to detect, since observations from a single site will typically not cover an entire orbit. A re-analysis of these data places an upper limit of $\sim 0.013$ mag on any reflection effect (M. Reed, private communication). Given the uncertainties involved in these calculations and comparisons, we do not feel confident completely ruling out an M3 dwarf companion at this stage, but instead suggest the white dwarf scenario is more likely.

Finally, using our high resolution UV spectra, we may be able to improve on the equatorial rotation velocity limits set by Heber et al. (2000) and therefore constrain the orbital inclination and companion mass. In the near UV our spectral resolution is almost a factor of two better than the spectra used by Heber et al., so we shifted each of the six spectra used to determine radial velocities to their rest wavelength and then averaged them. The spectrum was examined for metal lines in close blends that are clearly resolved (a difficult task due to the very large number of unresolved blends), and these lines were fit with a variety of values of $v \sin i$. We show the three Fe III lines chosen in Fig. 5, with three different rotation velocities $v \sin i=0,5$, and $10 \mathrm{~km} \mathrm{~s}^{-1}$. There is no clear difference between the $0 \mathrm{~km} \mathrm{~s}^{-1}$ (dashed) and $5 \mathrm{~km} \mathrm{~s}^{-1}$ curves (dotted), while $v \sin i=10 \mathrm{~km} \mathrm{~s}^{-1}$ (triple-dash-dotted curve) does not 


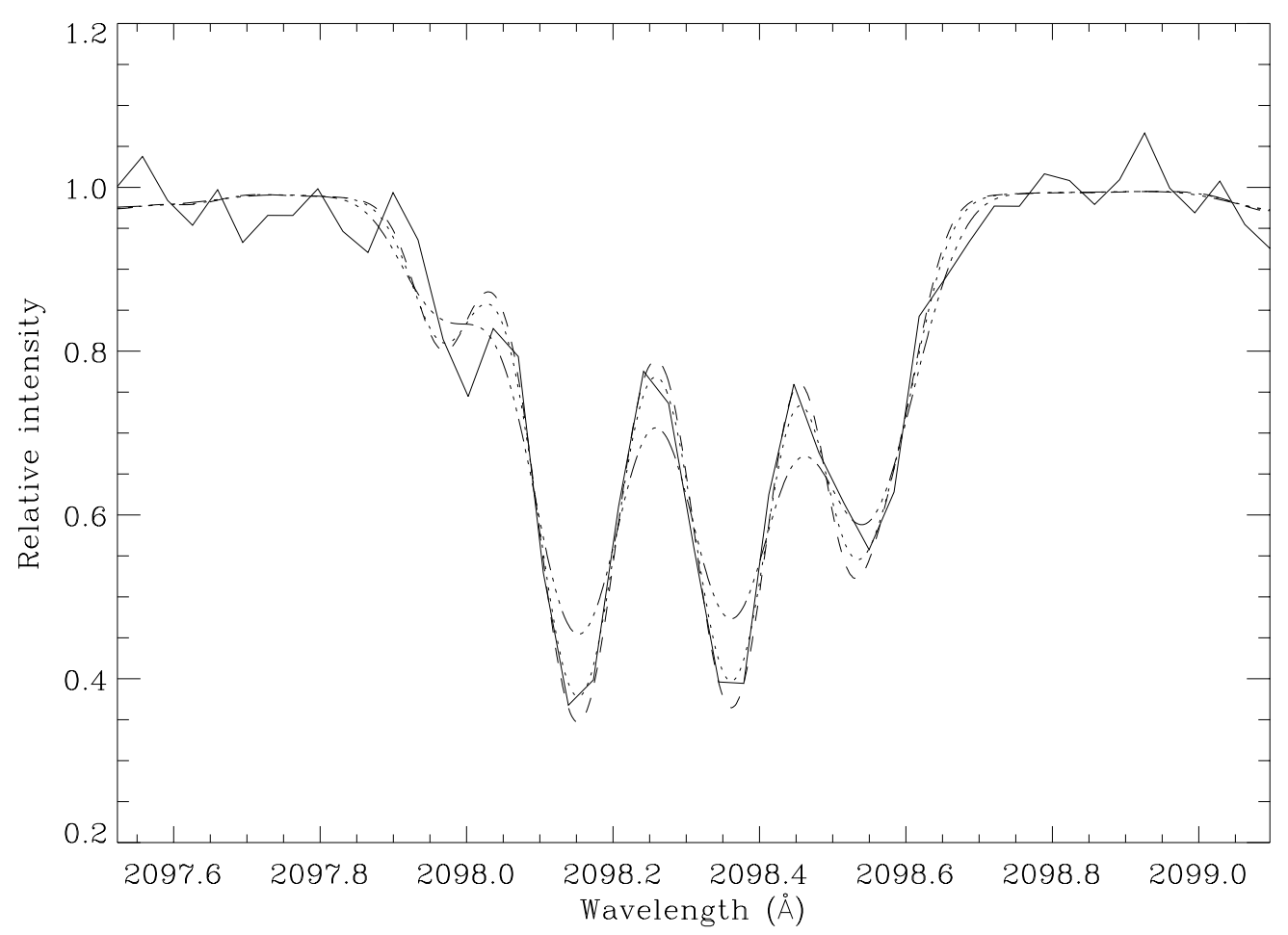

Fig. 5. Fit of $v \sin i$ to three Fe III lines. The line at $\sim 2098.5 \AA$ is blended with a Mn III line. The dashed line represents $v \sin i=0 \mathrm{~km} \mathrm{~s}^{-1}$, the dotted line is $v \sin i=5 \mathrm{~km} \mathrm{~s}^{-1}$, and the dash-triple-dot line is $v \sin i=10 \mathrm{~km} \mathrm{~s}^{-1}$. See the text for further details.

appear to fit. After a simple $\chi^{2}$ analysis, we find that $v \sin i=$ $4.8 \pm 1.2 \mathrm{~km} \mathrm{~s}^{-1}$. At a $3 \sigma$ level $v \sin i$ is constrained to less than $8 \mathrm{~km} \mathrm{~s}^{-1}$. Together with the upper limit of $5 \mathrm{~km} \mathrm{~s}^{-1}(3 \sigma)$ derived from the $\mathrm{Mg}$ II line in the optical (Heber et al. 2000), we consider $5 \mathrm{~km} \mathrm{~s}^{-1}$ as an upper bound the following discussion.

The rotation of Feige 48 is expected to be tidally locked to the orbit. In this case we can estimate the rotation velocity from the orbital period and the radius $\left(\sim 0.2 R_{\odot}\right)$ to be $26.9_{-1.6}^{+1.5} \mathrm{~km}^{-1}$ (this is coincidentally approximately the same as the orbital velocity). Comparison with the projected rotational velocity constrained above yields an upper limit to the inclination of the system of $i=11.4^{\circ}$. This is the same upper limit derived by Reed et al. (2004) and Reed (2001) when they combined the upper limit to the rotation velocity derived by Heber et al. (2000) and the rotation period derived from their asteroseismological analysis. From the mass function we derive a lower limit to the companion mass of $M_{2}=0.46 M_{\odot}$. This result means that the companion must be degenerate, and is most likely a white dwarf. Considering the orbital period and companion mass, the Feige 48 system should have gone through two common envelope phases, and therefore can be explained by the "second common envelope ejection channel" described by Han et al. (2003).

\section{Discussion and conclusions}

We have detected binary motion in the V361 Hya star Feige 48 with a period of $0.376 \mathrm{~d}$ and a velocity semi-amplitude of $28.0 \mathrm{~km} \mathrm{~s}^{-1}$. Combining multicolour photometry, the asteroseismological determination of the rotation period, and an upper limit of the equatorial rotation velocity, we conclude that the orbital inclination $i \leq 11.4^{\circ}$. This implies that the minimum mass of the companion is $0.46 M_{\odot}$; combining this with the lack of a reflection effect suggests the star must be a white dwarf.

Feige 48 is one of only two pulsating sdBs with a known white dwarf companion. Unlike KPD 1930+2752, the other system, it has a relatively simple pulsation spectrum and no (or very little) ellipsoidal distortion. This has allowed Reed (2001), Reed et al. (2004) and Charpinet et al. (2003) to match pulsation frequencies and constrain sdB evolution models. However, these studies assumed that Feige 48 evolved according to canonical EHB theory. While this is probably accurate since the star has undergone two phases of common envelope evolution, the conclusions of Han et al. (2003) suggest that investigating a wider mass range than previously may be warranted, with masses as low as $\sim 0.36 M_{\odot}$. In the case of single sdBs, which should be formed through the merger channel, masses in the range $0.4-0.7 M_{\odot}$ should be examined. The sdBs in longperiod orbits with main sequence companions (formed through the Roche lobe overflow channel) may fall in the $0.3-0.5 M_{\odot}$ range, and possibly even higher, although Han et al. find this to be unlikely. Enlarging the mass range investigated in this way may provide another useful test of the proposed formation models for sdBs.

These tests can only be carried out once the binary nature of the pulsating $\mathrm{sdBs}$ is known. Up to now only seven V361 Hya stars are confirmed binaries; four have F- or G-type main sequence companions (V361 Hya itself, PB 8783, EC 20117-4014 and EC 10228-0905), two have white dwarf companions (KPD 1930+2752 and Feige 48), and one is in an eclipsing binary system with an M5 main sequence 
star (PG 1336-018). That leaves around 25 pulsators with unknown status. The fraction of sdBs is binary systems is still under debate, however if we consider the best-fit simulation of Han et al. (2003), we find that $\sim 41 \%$ or $10-11$ of these stars should have either late-type main sequence or white dwarf companions. Lisker et al. (2004) compared their observations of $52 \mathrm{sdB}$ stars with the 12 simulation sets constructed by Han et al. (2003) using a simple statistical test. The simulation set they found to fit their observations differs from that found qualitatively by Han et al. and suggests that up to $\sim 53 \%$ or $\sim 13$ of the pulsating sdBs should have an unseen companion. High precision spectra are needed to observe short period systems such as Feige 48, so a survey with high spectral and temporal resolution is required.

Acknowledgements. We would like to thank Mike Reed and Horst Drechsel for useful discussions. S.J.O.T. is supported by the Deutsches Zentrum für Luft- und Raumfahrt (DLR) through grant No. 50-OR-0202. R.A.B. acknowledges support from the FUSE guest observer program (NAG 5-12196). This publication makes use of data products from the Two Micron All Sky Survey, which is a joint project of the University of Massachusetts and the Infrared Processing and Analysis Center/California Institute of Technology, funded by the National Aeronautics and Space Administration and the National Science Foundation.

\section{References}

Benjamin, R. A., Wakker, B. P., \& Richter, P. 2004, ApJ, in preparation

Bern, K., \& Wramdemark, S. 1973, Lowell Obs. Bull., 8, 1

Beuermann, K., Baraffe, I., \& Hauschildt, P. 1999, A\&A, 348, 524

Charpinet, S., Brassard, P., \& Fontaine, G. 2003, in Extreme Horizontal Branch stars and related objects, ed. P. Maxted (Kluwer), in press

Chayer, P., Fontaine, G., Fontaine, M., et al. 2003, in Extreme Horizontal Branch stars and related objects, ed. P. Maxted (Kluwer), in press
Cohen, M., Wheaton, W. A., \& Megeath, S. T. 2003, AJ, 126, 1090 D'Cruz, N. L., Dorman, B., Rood, R. T., \& O'Connell, R. W. 1996, ApJ, 466, 359

Dorman, B., Rood, R. T., \& O’Connell, R. W. 1993, ApJ, 419, 596

Fukugita, M., Shimasaku, K., \& Ichikawa, T. 1995, PASP, 107, 945

Green, E. M., Fontaine, G., Reed, M. D., et al. 2003, ApJ, 583, L31

Han, Z., Podsiadlowski, P., Maxted, P. F. L., \& Marsh, T. R. 2003, MNRAS, 341, 669

Han, Z., Podsiadlowski, P., Maxted, P. F. L., Marsh, T. R., \& Ivanova, N. 2002, MNRAS, 336, 449

Heber, U. 1986, A\&A, 155, 33

Heber, U., Reid, I. N., \& Werner, K. 2000, A\&A, 363, 198

Heber, U., Edelmann, H., Lisker, T., \& Napiwotzki, R. 2003, A\&A, 411, L477

Heber, U., Drechsel, H., Østensen, R., et al. 2004, A\&A, 420, 251

Iben, I. J., \& Tutukov, A. V. 1986, ApJ, 311, 753

Kilkenny, D. 2002, in IAU Coll. 185, Radial and Nonradial Pulsations as Probes of Stellar Physics, ASP Conf. Ser., 259, 356

Koen, C., O'Donoghue, D., Pollacco, D. L., \& Nitta, A. 1998, MNRAS, 300, 1105

Lisker, T., Heber, U., Napiwotzki, R., et al. 2004, A\&A, submitted

Maxted, P. F. L., Heber, U., Marsh, T. R., \& North, R. C. 2001, MNRAS, 326, 1391

Mengel, J. G., Norris, J., \& Gross, P. G. 1976, ApJ, 204, 488

Monet, D. G., Levine, S. E., Canzian, B., et al. 2003, AJ, 125, 984

Moos, H. W., Cash, W. C., Cowie, L. L., et al. 2000, ApJ, 538, L1

Morales-Rueda, L., Maxted, P. F. L., Marsh, T. R., North, R. C., \& Heber, U. 2003, MNRAS, 338, 752

Napiwotzki, R., Karl, C. A., Lisker, T., et al. 2004, in Extreme Horizontal Branch stars and related objects, ed. P. Maxted (Kluwer) [arXiv: astro-ph/0401201]

O'Toole, S. J., Heber, U., Chayer, P., et al. 2003, in Extreme Horizontal Branch stars and related objects, ed. P. Maxted (Kluwer) [arXiv: astro-ph/0309062]

Reed, M. D. 2001, Ph.D. Thesis, Iowa State University

Reed, M. D., Kawaler, S. D., Zola, S., et al. 2004, MNRAS, 348, 1164

Saffer, R. A., Bergeron, P., Koester, D., \& Liebert, J. 1994, ApJ, 432, 351 